\title{
A guide to OSCEs: my findings on examination
}

Clinical examination: a systematic guide to physical diagnosis. 7th ed. Nicholas J Talley, Simon O'Connor. Sydney: Churchill Livingstone Australia, 2013 (624 pp, \$125). ISBN 9780729541473.

WITH OVER 25 years of development and review contributing to Talley and O'Connor's Clinical examination, it is no wonder that the educational guide, now in its 7th edition, has become an essential reference for medical students.

While maintaining its dependable content, the ne $\mathrm{w}$ edition has added depth, particularly regarding investigations useful for the diagnosis and ongoing evaluation of common differential diagnoses.

Examples include indications for and interpretation of echocardiograms, abdominal computed tomography scans and lumbar punctures. This is consistent with a clear shift towards supporting students in their preparation for assessment, which involves more than physical examination.

The exam focus is substantiated by the addition of "OSCE (objective structured clinical examination) revision topics", "T\&O'C essentials" and a far more reader-friendly format. While the book preserves its

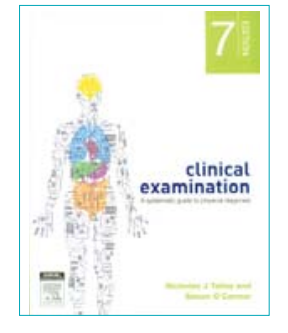

system-based structure, the clear division of content ensures information is significantly more accessible.

The guide takes a further step towards preparing students for their future medical practice, with additional chapters on the assessment of the geriatric patient, of the acutely ill patient and of death. While the latter begins to extend beyond the immediate scope of a student, the topics are only covered superficially and, at most, would serve as a starting point for supplemental reading in emergency and end-of-life care. It is important to bear in mind that these topics are not a pressing concern to students in their current clinical roles and, as such, would not justify significantly greater coverage.

There is no doubt that Clinical examination is the cornerstone reference for medical students studying the skill of physical diagnosis. The systematic guide also provides experienced physicians with a reliable and thorough resource on examination findings and patient interactions. It is highly recommended to all those seeking to improve their understanding of, or requiring succinct templates for, structured doi: 10.5694/mjal4.01043 